\section{Discrimination of restrictions in sequentially-encoded auditory displays: Block designs*}

\author{
IRWIN POLLACK $\dagger, \dagger \dagger$ \\ The University of Michigan, Ann Arbor, Michigan 48104
}

Sequential constraints, in the form represented by equal frequencies within blocks, were imposed upon finite-state statistical generators. The sequences were encoded in the form of interval-coded pulse trains and were transduced to sound by earphones. In discrimination tests, interval thresholds between the finite states provided a measure of the relative discriminability between different sequential constraints. These thresholds are shown to be quantitatively related to the difference in uncertainty in specification of the sequences. To a first approximation: Equal interstate interval thresholds are associated with equal differences in uncertainty.

An efficient procedure for examining the sensitivity of Ss to statistical constraints is obtained through the use of interval-coded auditory displays (Pollack, $1967,1968 \mathrm{a}, \mathrm{b})$. Specifically, finite-state sequences may be constructed to reflect a wide variety of statistical generators, and their outputs may be efficiently presented as auditory displays at rates to nearly 10,000 samples per second. Depending upon the frequency range of the signals, the auditory system employs a variety of processing mechanisms for the treatment of such data.

The present paper considers the detection and the relative discrimination of sequential constraints represented by equal frequencies within defined blocks. The aim of the study was to search for a relation between the ease of discriminability of various sequences and an independent measure of their degree of sequential constraint.

\section{METHOD}

Finite-state sequences were generated by the procedure illustrated in Table 1 . The number of different states, $m$, and the length of a given block, B, determined the number of occurrences of each state within each block. Each state occurred $\mathrm{B} / \mathrm{m}$ limes. The first three lines illustrate the effect of different block lengths with a constant number of states, 2. (The commas are presented 10 aid in the visual identification of block length; there was no marker in the

\footnotetext{
* This research was supported by National Science l'oundation Grant GB 6148 .

Hailing address: Mental Health Research Institute, The Universily of Michigan. Ann Arbor, Michigan 48104.

TiThe wrier is deeply indebted to a patient crew of listeners, to Mrs. Kathryn Weidner who supervised the testin" program, to Mr. Robert thich for the procesing of the expermentul rescilts, and to Mr. Lon Radin who wrote the computer experimental program.
}

auditory pulse trains.) It is noted that the maximum possible run length is twice the number of occurrences of each state within each block. The last three lines illustrate the effect of a different number of states, $\mathrm{m}$, with a constant block length, 8 . Within each block, the order of occurrence of all states was scrambled. The entire process was replicated $N / B$ times to provide the sequence of $N$ items in length. For example, with $m=2, B=6, N=600$; a list of three representations of each of 1 wo states was successively scrambled 100 times to provide a sequence of 600 items.

Two-state sequences were presented 10 listeners by translating the state designations to intervals between successive pulses, and then transducing the pulses to sound by earphones (Koss PRO-4). For example, if we represent the 0 state by an interpulse interval of $0.4 \mathrm{msec}$, and the 1 state by an interpulse interval of $0.6 \mathrm{msec}$, the sequence 0110 would be translated into: pulse: a wait of $0.4 \mathrm{msec}$; pulse; a wait of 0.6 msec; pulse; a wait of $0.6 \mathrm{msec}$; pulse; a wait of $0.4 \mathrm{msec}$; and pulse. In this sequence the average interpulse interval,

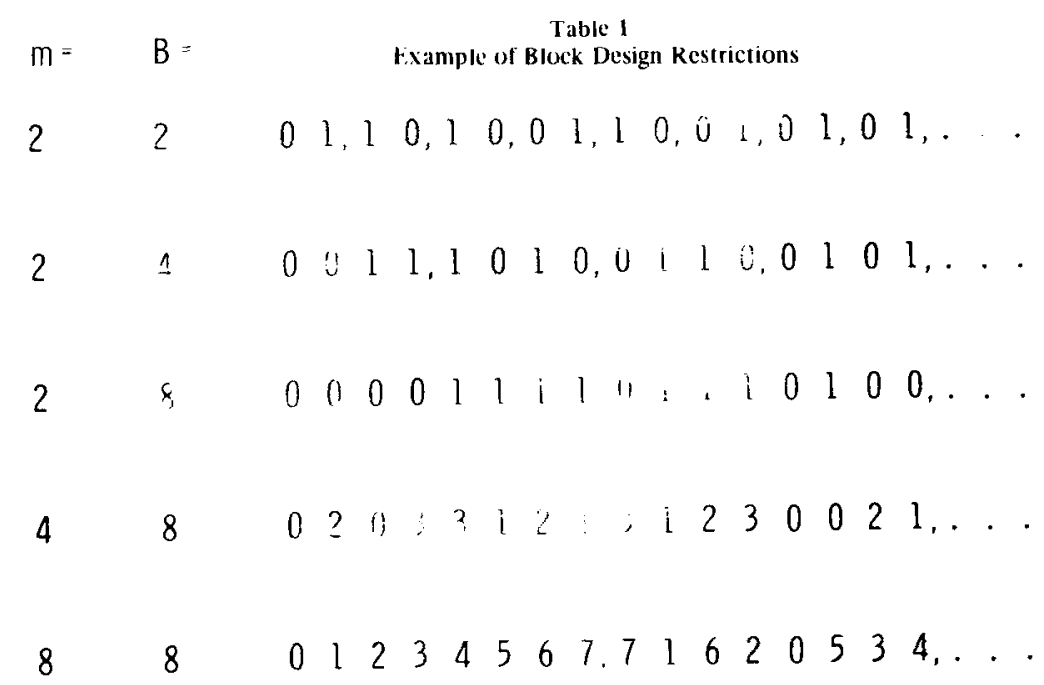

$\mathrm{IPI}_{\mathrm{c}}$, is $0.5 \mathrm{msec}$; the difference between the two states, DIPI, is $0.2 \mathrm{msec}$, or $40 \%$ of the mean interval.

The listener heard four pulse trains: three generated with the smaller block length, $S$, and one generated with the larger block length, L. His task was to indicate which one of the four pulse trains sounded different from the other three. For example, with $\mathrm{m}=2, \mathrm{~S}=6, \mathrm{~L}=12$, $\mathrm{N}=600$; three of the pulse trains represented 100 successive independent scramblings of a list of three representations of each of two states; one of the pulse trains represented 50 successive independent scramblings of a list of six representations of each of the two states. Within all four sequences, IPI $I_{c}$ and DIPI were identical, i.e., the states were marked in the same way, irrespective of the block length.

One nonobvious assumption is made. An ordinal measure of the relative discriminability among the sequences is provided by the interstate threshold difference, DIPI. Consider the extreme case where the two states are represented by the same interpulse interval. In this case, all sequences are identical since the two states are interchangeable. While the initial assumption may be reasonable in the extreme case, what kind of evidence can be marshaled for the assumption when the intervals are not identical? The first line of evidence is that in a previous, related study corresponding answers were obtained, on the one hand, by fixing DIPI and varying sequence parameters and, on the other hand, by fixing sequence parameters and varying DIPI. In each case, DIPI must be increased as the sequential parameters approach each other (Pollack, 1968a). The second line of evidence is that DIPI was controlled by an adaptive stimulus-programming procedure in the present tests and the method works. Specifically, if the listener voted correctly

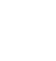




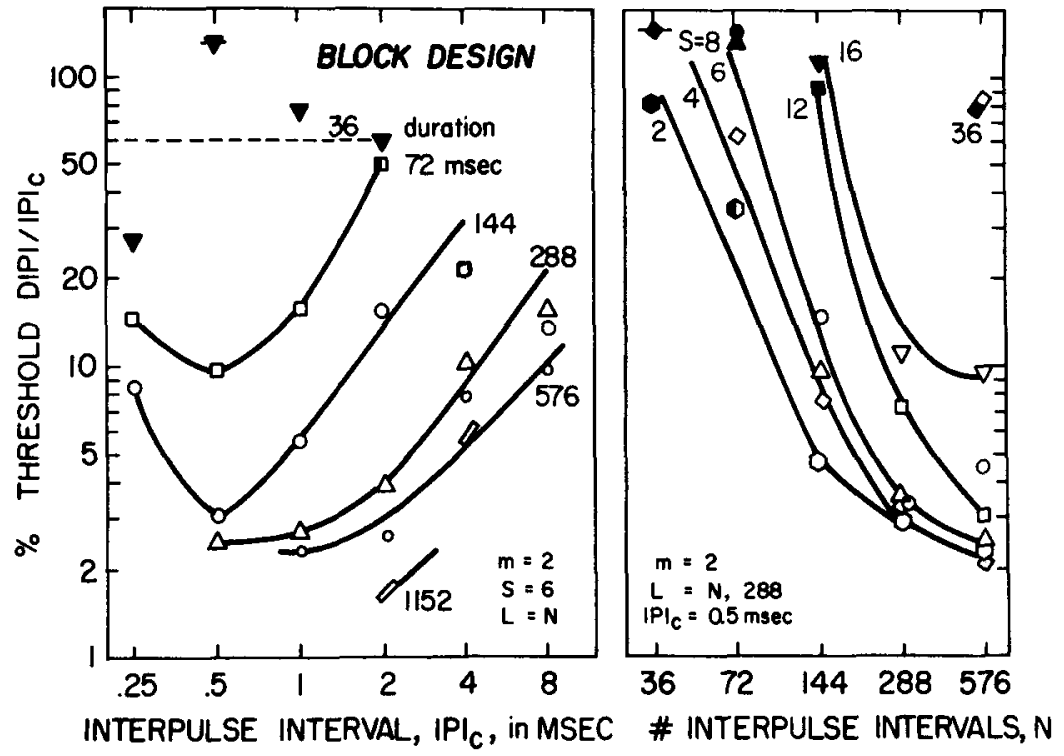

for the signal with the block length, L, from among the four pulse trains, DIPI was decreased; if he voted incorrectly, DIPI was increased. We thus aimed for $50 \%$ correct in the four-interval forced-choice (4IFC) procedure. If DIPI were irrelevant to the task, only random convergence would have been obtained. This was not the case.

Unless specified otherwise, the following parameters were employed: $\mathrm{N}=600$ (or a near integer of $\mathrm{B}$ near 600$) ; \mathrm{m}=2$; $\mathrm{IPI}_{\mathrm{c}}=0.5 \mathrm{msec}$; and pulse duration of 10 microseconds. Limits upon the testing conditions imposed by the program were: $\mathrm{L}=300 ; \mathrm{N}=625 ;$ maximum DIPI = $2\left(\mathrm{IPI}_{\mathrm{c}}-.131 \mathrm{msec}\right) /(\mathrm{m}-1)$. When the sought-for DIPI exceeded the limit, an additional observation was presented at the limiting condition of DIPI. If the listener responded incorrectly on that observation, the trial was terminated. Each of 15 listeners contributed at least two thresholds under each of 277 experimental conditions. Median thresholds, which include those trials that exceeded the limits of the program, are reported. Results are coded in the graphs as follows: Open points imply that the percentage of terminated trials was $<10 \%$; half-filled points imply $10 \%-20 \%$ terminated trials; filled points imply $20 \%-50 \%$ terminated trials; filled points with a horizontal bar imply $>50 \%$ terminated trials.

The unit of temporal control for DIPI was $3 / 8$ microsecond. The minimum threshold was one-half of the unit of temporal control. Successive pulse trains were separated by $0.5 \mathrm{sec}$. Successive observations were separated by $1 \mathrm{sec}$ following the listener's response. Listening was carried out at a comfortable listening level.

Ss were university music majors or students with a musical background. They may be considered experienced; a majority served in comparable tests over more than one semester, $5 \mathrm{~h}$ per week.

Each point consists of the median of at least two thresholds contributed by each of 15 listeners. Stimulus generation, execution of the adaptive stimulus programming procedure of variable step size, and optimal termination of the adaptive procedure were accomplished by the PEST procedure (Taylor \& Creelman, 1967), implemented by a PDP. 8 computer (Digital Equipment Corporation), programmed by Mr. Lon Radin.

\section{RESULTS}

(1) What is the Effect of the Sample Presentation Rate and the Number of Intervals upon the Discrimination of Block Length?

The left side of Fig. I considers the discriminability of two-state sequences with a smaller block length of 6 from a larger block length equal to the total length of the sequence. The different sample presentation rates are represented along the abscissa in terms of the average interpulse interval. The duration of the pulse trains is the parameter. The ordinate is the threshold magnitude of DIPI, relative to the mean interval.

Lowest thresholds are obtained with shorter interpulse intervals for pulse trains of a constant duration. Maximum sensitivity is obtained in the vicinity of $0.5 \mathrm{msec}$, or 2,000 samples per second. Subsequent tests will therefore employ an average interpulse interval of $0.5 \mathrm{msec}$.

The right side of Fig. I considers the discriminability of two-state sequences with several smaller block lengths, $S$, from a larger block length, L, limited only by the length of the sequence. Discrimination thresholds fall with a greater number of
Fig. 1. Interstate discriminability thresholds as a function of sample presentation rate (left section) and of number of interpulse intervals (right section). Each point of Fig. 1 and subsequent figures represents the median of at least two thresholds contributed by each of 15 listeners. Points are increasingly filled to reflect an increasing proportion of terminated trials (see text).

intervals. The improvement in thresholds is related, in part, to the number of blocks, but is also related to the total number of interpulse intervals. Discrimination tends to improve with the number of intervals for a constant number of blocks (up to a limit). For example, the following all employ 18 blocks: $\mathrm{N}=36, \mathrm{~S}=2 ; \mathrm{N}=72$, $S=4 ; N=144, S=8 ; N=288, S=16$. There is a tenfold range in DIPI thresholds over these conditions, al though the number of blocks was held constant.

(2) How is Discrimination Dependent upon Block Length?

Figure 2 presents the results of tests with a constant number of states, $m=2$, as a joint function of the smaller block length (parameter) and the larger block length, $L$ (abscissa). For example, a threshold DIPI of about $5 \%$ is required for $\mathrm{S}=2$ and $L=4$. The $X s$ represent thresholds for a constant $\mathrm{L} / \mathrm{S}$ ratio of 2.0 , i.e., $\mathrm{L}=4, \mathrm{~S}=2$; $\mathrm{L}=8, \mathrm{~S}=4 ; \ldots ; \mathrm{L}=64, \mathrm{~S}=32$.

For a given $S$, thresholds decrease as the larger block size, $\mathrm{L}$, is increased. A range of shorter block lengths $(2 \leqslant S \leqslant 8)$ yield equivalent thresholds when paired with a

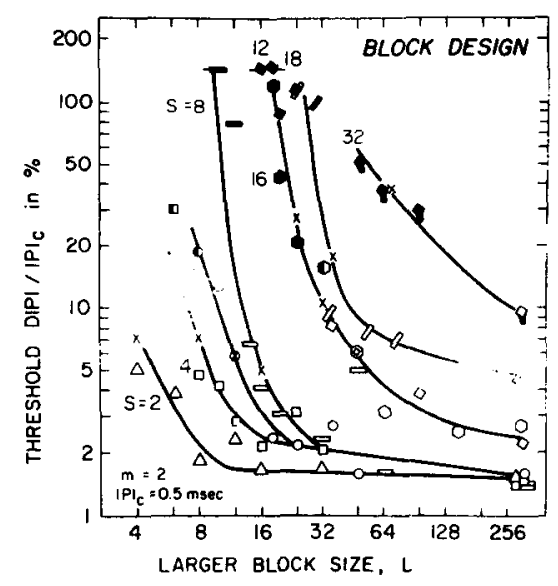

Fig. 2. Interstate discriminability thresholds as a function of block length. The larger block length, $L$, is represented on the abscissa; the smaller block length, $S$, is represented as the parameter. The Xs reflect thresholds at a constant $L / S$ ratio of 2.0. 


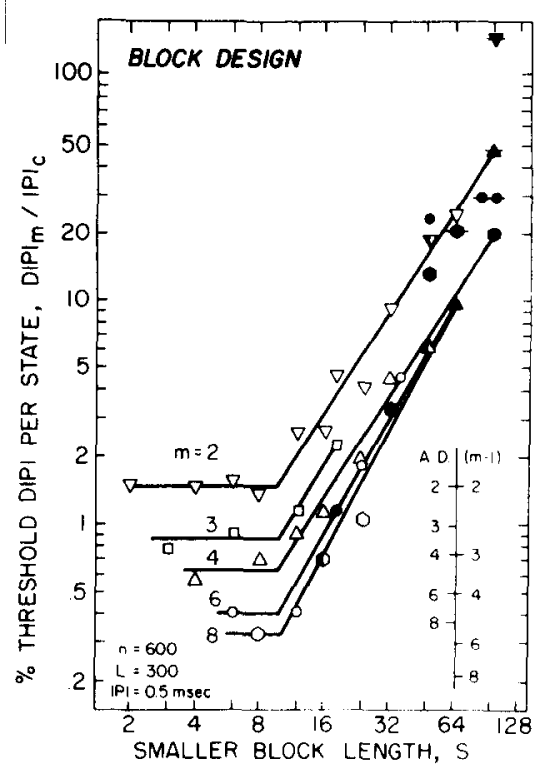

Fig. 3. Interstate discriminability thresholds as a function of the number of states. The abscissa is the shorter block length, $S$. The insert represents the expected spacing among the curves on the assumption of a constant average deviation among the states (A.D.) and a constant range of intervals between extreme states $(m-1)$.

large block length $(\mathrm{L}=300)$. For the same range of $\mathrm{S}$ block lengths, the major change in DIPI thresholds occurs by an $\mathrm{L} / \mathrm{S}$ ratio of about 6; and nearly equal DIPI thresholds are obtained for a constant L/S ratio, as represented by the Xs. Larger DIPI thresholds are associated with $12 \leqslant S \leqslant 32$. Indeed, consistent discrimination, i.c., $<20 \%$ terminated trials, is associated with $\mathrm{S}=32$ only for the condition $\mathrm{L}=300$.

(3) What is the Effect of the Number of Different Elements on Discrimination Thresholds?

Figures 3 and 4 extend the analysis to a greater number of states. One problem in comparing thresholds across a different number of states is that there exists several excellent alternatives for expressing the dependent variable: DIPI $m$ (the difference in interpulse interval between adjacen1 states, otherwise listed as DIPI); $\mathrm{DIPI}_{R}$ (the range of interpulse intervals associated with the extreme states); or DIPI the average deviation of interpulse intervals

Fig. 4. Interstate discriminability thresholds as a joint function of the number of states and block length. Each section represents a constant shorter block length. The abscissa represents the $L / S$ ratio, the ratio of the larger to the smaller block length. about the average or center interpulse interval, $\left.I P I_{c}\right)$.

The dependent measure of performance chosen in Fig. 3 is DIPI ${ }_{m}$. The insert graph indicates the spacing among the curves that would have been expected on the basis of a constant DIPI (left insert marked A.D.) and of a constant range of interpulse intervals (right insert marked $\mathrm{m}-1$ ), where $(m-1) \cdot D^{-P I} I_{m}=D_{P I P I}$

The constancy of DIPI in Fig. 3 for short block lengths parallels the finding of Fig. 2; when short $(2 \leqslant S \leqslant 8)$ block lengths are compared with an extreme block length (300), threshold DIPI is independent of $\mathrm{S}$ for a constant number of states. The inserts of Fig. 3 suggest that the spacing across $\mathrm{m}$ is appropriate to a compromise between a constant DIPI and a constant $\mathrm{DIPI}_{\mathrm{R}}$. The appropriate spacing across $m$ at the larger block lengths, on the right side of the graph, is difficult to discern because of the large proportion of terminated trials.

Noteworthy also is that discrimination tends to break down (in terms of the proportion of terminated trials) at somewhat shorter block lengths for the larger number of states than for the smaller number of states. In any event, it was difficult in the present tests to discriminate consistently with the smaller block length, $\mathrm{S}$, greater than 32, irrespective of the number of possible states.

Results comparable with the discrimination tests of Fig. 2 were also obtained for $m \geqslant 2$. A sampling of these results is presented in Fig. 4 for a small range of $\mathrm{L} / \mathrm{S}$ ratios. The parameter on the curves is the number of states; the separate sections represent successively longer $S$ block lengths. The dependent performance measure is $\operatorname{DIPI}_{R}$, the range of threshold interpulse intervals. Conditions with $\mathrm{L} / \mathrm{S}<2.0$ were also run, but they typically yielded a high proportion of terminated trials.

In terms of $\mathrm{DIPI}_{R}$, there is a consistent ordering for small $S, S \leqslant 8$, in favor of a smaller number of states. In the discrimination of, for example, a block length of 6 from a block length of 12 $(S=6 ; L / S=2)$, lowest thresholds are obtained with two-state sequences (three occurrences per block of each of two states vs six occurrences per block); intermediate thresholds with three-state sequences (two occurrences per block of each of three states vs four occurrences); and highest thresholds with six-state sequences (one occurrence of each of six states vs two occurrences). The strong ordering in favor of the smaller number of states becomes weakened for intermediate $S, 12-16$. There may be a suggestion (right section) that the strong ordering may be reestablished at still larger $\mathrm{S}$ values.

\section{DISCUSSION}

The overwhelming result is that discrimination thresholds decrease as the block lengths, $\mathrm{S}$ and $\mathrm{L}$, diverge, for a fixed number of states. We now seek a quantitative description of the effect of the

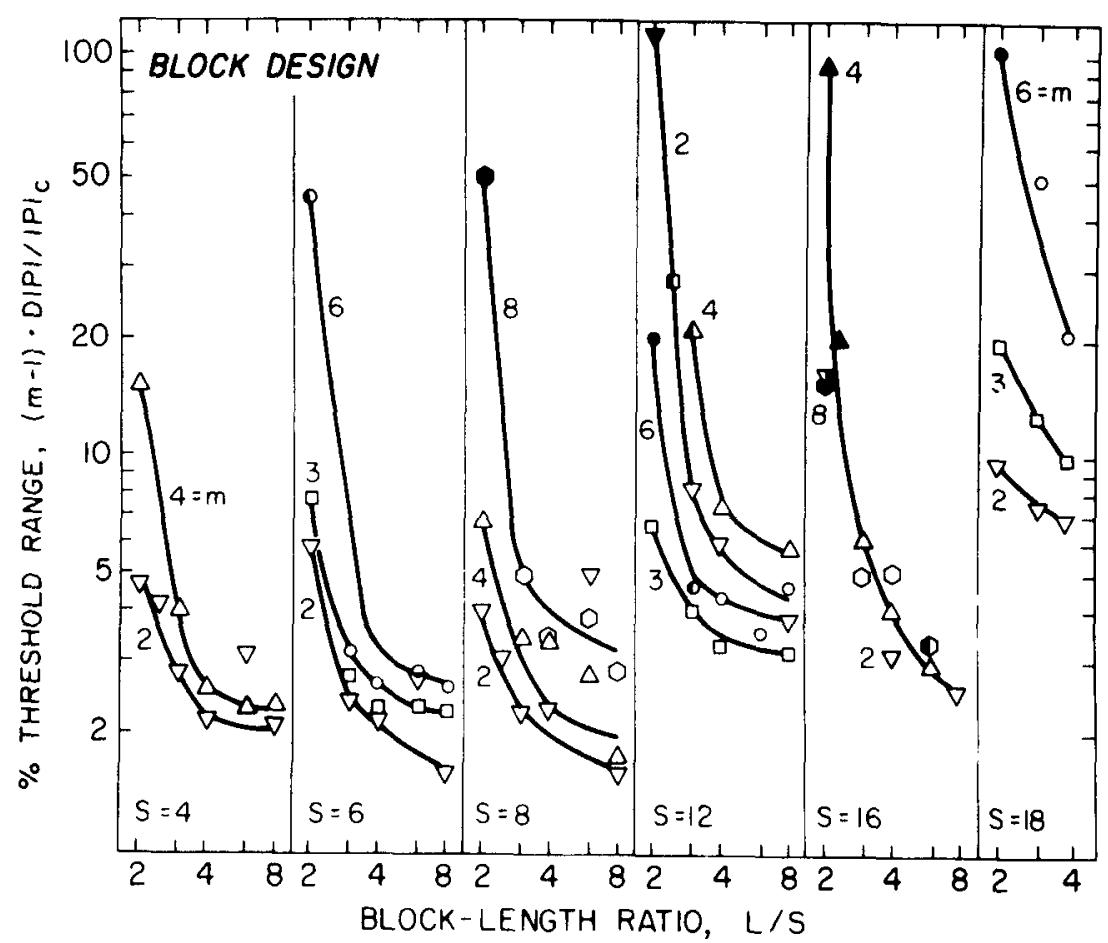




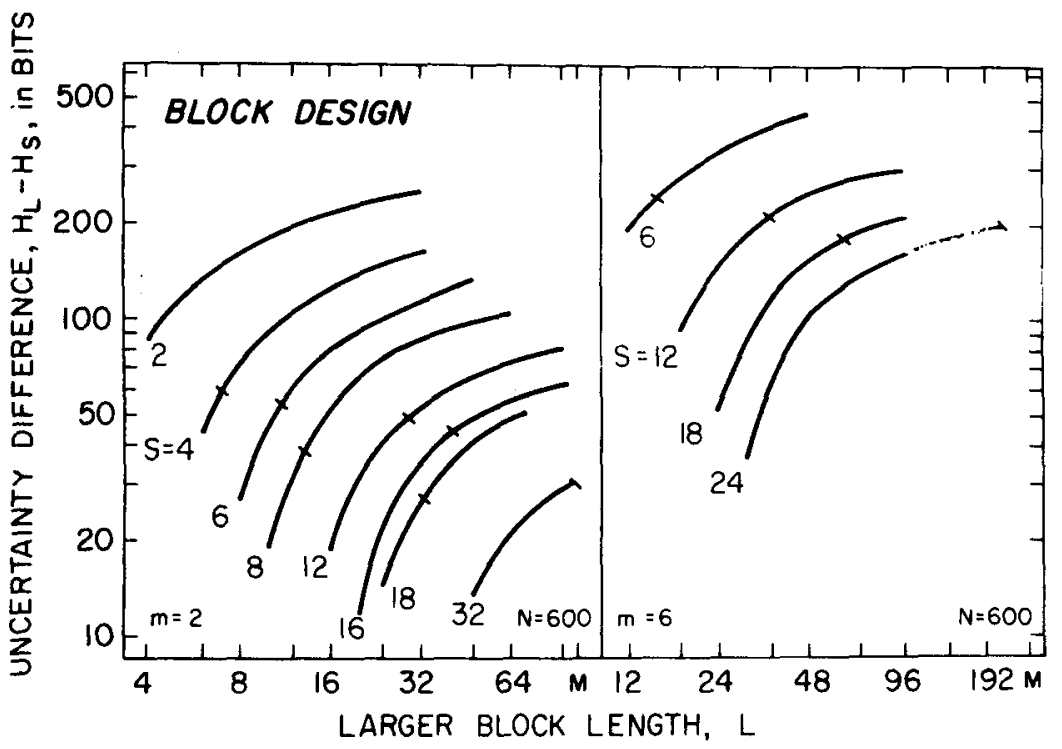

sequential restrictions represented by block length. For each pair of sequences, two measures of discriminability were obtained. Threshold DIPI serves as an inferred measure of discriminability, based upon the listening tests. (No assumption was made about the scaling properties of the measure other than a monotonic relationship). The difference in informational uncertainty associated with the $\mathrm{L}$ and $\mathrm{S}$ sequences serves as an independent measure of the relative discriminability of the sequences. The behavior of the uncertainty difference, as a function of $S$ and $L$, is illustrated in Fig. 5. The left section presents calculations for $\mathrm{m}=2$; the right section presents calculations for $m=6$. Each oblique dash signifies the region where the proportion of terminated trials increases abruptly with in the experiment. In terms of this crude measure, ease of discrimination is related to the uncertainty difference between the sequences: An uncertainty difference of about 40 bits is required for $\mathrm{m}=2$; an uncertainty difference of about 200 bits is required for $m=6$. Stated otherwise, the sequential restrictions imposed upon six-state sequences must differ more than those imposed upon two-state sequences for an equivalent level of discrimination.

The relation between the calculated uncertainty measure and the experimental

Fig. 6. Comparison between interstate discriminability thresholds and the difference in informational uncertainty. The left section represents conditions with $m=2, \quad N=600$. The right section represents conditions with $S=6, m=2$. trials) to open figures $(\leqslant 10 \%$ terminated trials) as the uncertainty difference is increased. In the same manner, the average DIPI decreases smoothly. Moreover, the
Fig. 5. Difference in informational uncertainty for selected block lengths. The oblique dash represents the approximate region where the proportion of terminated trials increases abruptly.

two average functions are nearly identical.

In summary, the characterization of the sequences in terms of differences in uncertainty provides a general descriptive metric over a wide range of block lengths. This metric is, in turn, related to an experimental measure of the relative discriminability between the sequences, DIPI. Consistent relationships between DIPI and uncertainty are achieved for a given number of states, although a single invariant relationship is not observed across a different number of states.

\section{REFERENCES}

POLLACK, I. Discrimination of changes in the structure of auditory Markov scquences. Proccedings, 75th Annual Convention of the American Psychological Association, 1967, 2, 35-36.

POLLACK, 1. Periodicity discrimination for auditory pulse trains. Journal of the Acoustical Society of America, 1968a, 43, 1113-1119.

POLLACK, 1. Discrimination of changes in Markov sequences with the first $\mathrm{N}$-th orders statistically independent. Proceedings, 76th Annual Convention of the American Psychological Association, 1968b, 125-126.

TAYLOR, M., \& CRIELMAN, D. PEST Efficient cstimates on probability functions. Journal of the Acoustical Socicty of America, $1967,41,782-787$.

(Accepted for publication May 15, 1970.)

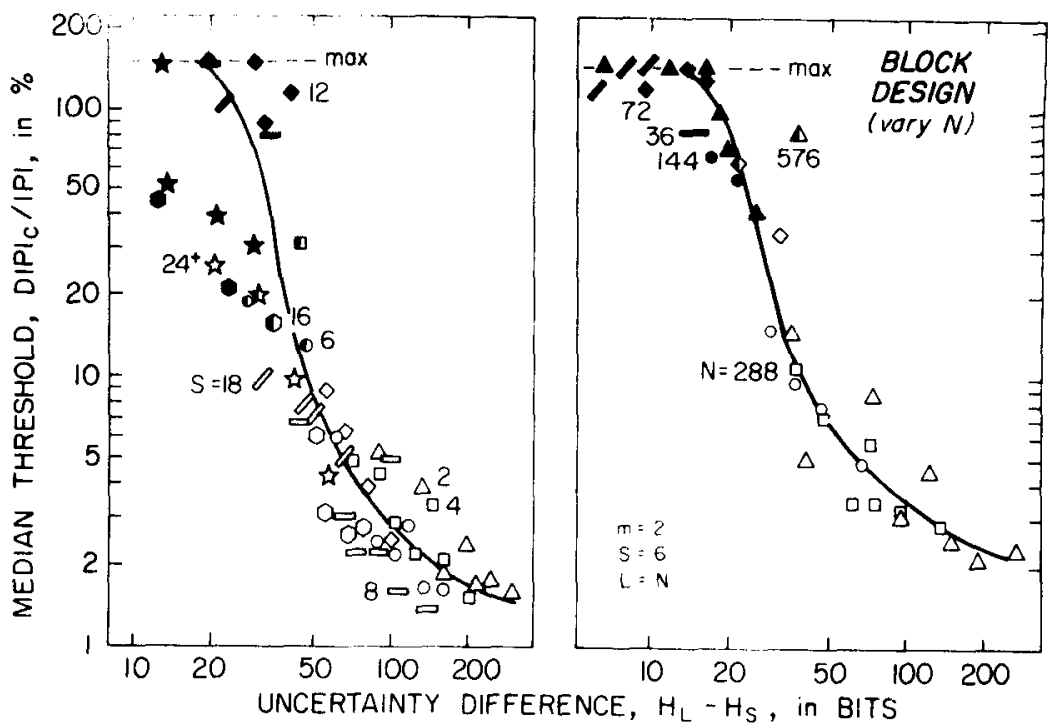

